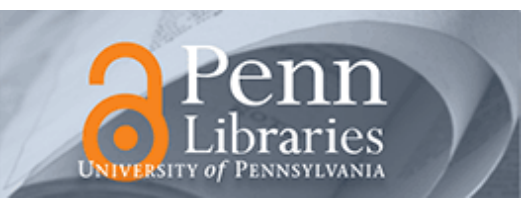

University of Pennsylvania

ScholarlyCommons

\title{
Subwavelength, Compact, Resonant Patch Antennas Loaded With Metamaterials
}

\author{
Andrea Alù \\ University of Pennsylvania \\ Filiberto Bilotti \\ University of Roma Tre \\ Nader Engheta \\ University of Pennsylvania, engheta@seas.upenn.edu \\ Lucio Vegni \\ University of Roma Tre
}

Follow this and additional works at: https://repository.upenn.edu/ese_papers

Part of the Electrical and Computer Engineering Commons

\section{Recommended Citation \\ Andrea Alù, Filiberto Bilotti, Nader Engheta, and Lucio Vegni, "Subwavelength, Compact, Resonant Patch Antennas Loaded With Metamaterials", . January 2007.}

Copyright 2007 IEEE. Reprinted from IEEE Transactions on Antennas and Propagation, Volume 55, Issue 1, January 2007, pages 13-25.

This material is posted here with permission of the IEEE. Such permission of the IEEE does not in any way imply IEEE endorsement of any of the University of Pennsylvania's products or services. Internal or personal use of this material is permitted. However, permission to reprint/republish this material for advertising or promotional purposes or for creating new collective works for resale or redistribution must be obtained from the IEEE by writing to pubs-permissions@ieee.org. By choosing to view this document, you agree to all provisions of the copyright laws protecting it.

This paper is posted at ScholarlyCommons. https://repository.upenn.edu/ese_papers/217

For more information, please contact repository@pobox.upenn.edu. 


\title{
Subwavelength, Compact, Resonant Patch Antennas Loaded With Metamaterials
}

\begin{abstract}
We analyze the matching and radiation properties of subwavelength resonant patch antennas filled with double-negative, double-positive, and/or single-negative metamaterial blocks. Analyzing the theoretical limits inherently present when loading such common radiators with metamaterials, we show how these configurations may exhibit in principle an arbitrarily low resonant frequency for a fixed dimension, but they may not necessarily radiate efficiently when their size is electrically small. However, interesting possibilities are suggested to overcome these limitations by employing circular or more complex patch geometries in order to select specific modes that, when appropriate loading ratios between the filling materials are chosen, also ensure radiation performance comparable qualitatively with a regular patch radiator of standard dimensions. Realistic numerical simulations, considering material dispersion, losses and the presence of the antenna feed are presented, showing how a practical realization is foreseeable. This may open novel venues in the design of small-scaled radiators with enhanced performance, which is of interest for many applications.
\end{abstract}

\section{Keywords}

metamaterials, miniaturized antennas, patch antennas

Disciplines

Electrical and Computer Engineering

\section{Comments}

Copyright 2007 IEEE. Reprinted from IEEE Transactions on Antennas and Propagation, Volume 55, Issue 1, January 2007, pages 13-25.

This material is posted here with permission of the IEEE. Such permission of the IEEE does not in any way imply IEEE endorsement of any of the University of Pennsylvania's products or services. Internal or personal use of this material is permitted. However, permission to reprint/republish this material for advertising or promotional purposes or for creating new collective works for resale or redistribution must be obtained from the IEEE by writing to pubs-permissions@ieee.org. By choosing to view this document, you agree to all provisions of the copyright laws protecting it. 


\title{
Subwavelength, Compact, Resonant Patch Antennas Loaded With Metamaterials
}

\author{
Andrea Alù, Student Member, IEEE, Filiberto Bilotti, Senior Member, IEEE, Nader Engheta, Fellow, IEEE, and \\ Lucio Vegni, Member, IEEE
}

\begin{abstract}
We analyze the matching and radiation properties of subwavelength resonant patch antennas filled with double-negative, double-positive, and/or single-negative metamaterial blocks. Analyzing the theoretical limits inherently present when loading such common radiators with metamaterials, we show how these configurations may exhibit in principle an arbitrarily low resonant frequency for a fixed dimension, but they may not necessarily radiate efficiently when their size is electrically small. However, interesting possibilities are suggested to overcome these limitations by employing circular or more complex patch geometries in order to select specific modes that, when appropriate loading ratios between the filling materials are chosen, also ensure radiation performance comparable qualitatively with a regular patch radiator of standard dimensions. Realistic numerical simulations, considering material dispersion, losses and the presence of the antenna feed are presented, showing how a practical realization is foreseeable. This may open novel venues in the design of small-scaled radiators with enhanced performance, which is of interest for many applications.
\end{abstract}

Index Terms-Metamaterials, miniaturized antennas, patch antennas.

\section{INTRODUCTION}

$\mathbf{N}$ OWADAYS the demand for compact radiators with sufficiently high gain is rapidly increasing in many application areas. In particular, modern wireless telecommunication systems and space communications require compact antennas with high gain, which become even more relevant requirements when the radiating elements have to be combined in large antenna arrays for satellites, space vehicles, airplanes, etc.

Microstrip patch antennas, due to their inherent capabilities (mainly low cost, low weight and low profile) are widely used in those setups (see, e.g., [1] and [2]). Even though such antennas are very thin compared to the operating wavelength $(0.05-$

Manuscript received November 2, 2005; revised June 22, 2006. The work of L. Vegni and F. Bilotti was supported in part by the European Commission 6th Framework Programme under Grant NMP3-CT-2005-500252, the work of N. Engheta was supported by the U.S. Defense Advanced Research Projects Agency (DARPA) under Grant HR0011-04-P-0042, and the work of A. Alù was supported in part by the 2004 SUMMA Graduate Fellowship in Advanced Electromagnetics.

A. Alù is with the Department of Applied Electronics, University of Roma Tre, 00185 Roma, Italy and also with the Department of Electrical and Systems Engineering, University of Pennsylvania, Philadelphia, PA 19104 USA (e-mail: alu@uniroma3.it, andreaal@ee.upenn.edu).

F. Bilotti and L. Vegni are with the Department of Applied Electronics, University of Roma Tre, 00185 Roma, Italy (e-mail: bilotti@uniroma3.it; vegni@uniroma3.it).

N. Engheta is with the Department of Electrical and Systems Engineering, University of Pennsylvania, Philadelphia, PA 19104 USA (e-mail: engheta@ee. upenn.edu).

Digital Object Identifier 10.1109/TAP.2006.888401
$0.01 \lambda)$ in their cross section, however, still their transverse dimensions cannot be made arbitrarily short, since a regular patch antenna resonates at a given frequency when its linear transverse dimension is of the order of half wavelength.

The interest in overcoming this limitation represents one of the main challenges for antenna designers. Over the years, indeed, several techniques have been proposed in order to squeeze the resonant dimensions of patch radiators, while maintaining their other radiation features. In this sense, the use of lumped reactive loads, slots on the patch surface [3], shorting pins [4], or high permittivity dielectrics may effectively lower the resonant frequency for a fixed dimension of the patch [1], [2]. However, the radiation pattern, the cross-polarization levels and the radiation efficiency may be worsened by the presence of such elements or by the excitation of surface waves.

The use of artificial materials and surfaces, properly engineered to improve some prescribed antenna features (impedance matching, gain, bandwidth, efficiency, front-to-back ratio, etc.) may represent a novel way of overcoming the limitations shown by some of the well known techniques for reducing the antenna size [5], [6]. In particular, the recent advances in the fabrication processes of ceramic oxide materials with high/low dielectric constants make the material tapering an effective technique to improve the performances of miniaturized antennas [7]. The possibility of material design, in fact, offers new degrees of freedom for the antenna designer. Several examples of compact radiators have been recently proposed by Volakis and his group [6], [8], exploiting properly textured engineered materials. The same group has also proposed the use of magnetic photonic crystals (MPC) that seems to be a promising avenue for achieving patch antenna miniaturization [9]. Other interesting possibilities to miniaturize patch antennas through the use of magnetodielectric materials and metasurfaces have also been recently proposed by Mosallaei and Sarabandi [10], [11].

In this context of novel artificial materials an important role may be played by metamaterials, which, due to their interesting anomalous electromagnetic features, have attracted a great deal of attention in recent years for several electromagnetic applications (see, e.g., [12]). Interest has been focused in particular on the atypical wave interaction of metamaterials with anomalous electromagnetic constitutive parameters, and in particular with negative real part of their permittivity [ $\epsilon$-negative ENG)], of their permeability $[\mu$-negative $(\mathrm{MNG})]$ or with both these quantities being negative [double-negative (DNG)] in a specific frequency range. Among the many applications, their use has been proposed to overcome the diffraction limit in various configurations. The phase compensation properties of DNG metamaterials may allow synthesizing subwavelength cavity resonators 
[13], waveguides [14] and scatterers [15] with resonant properties essentially independent on their effective physical size. In the quasi-static limit, when the retardation effects are negligible due to the small dimensions of such components and only one of the two constitutive parameters interact with the field depending on its polarization, even single negative ( $\mathrm{SNG}$ ) materials, i.e., ENG or MNG, may be utilized to achieve similar effects.

Such subwavelength resonances may be applied also to antenna configurations. We have recently proposed their utilization in planar [16] or cylindrical [17] subwavelength leaky-wave antenna configurations and for enhancing the radiation from subwavelength apertures in perfectly conducting screens [18], [19]. The use of ENG or DNG covers to enhance the radiation and matching properties of short electric dipoles has also been proposed by Ziolkowski and his group (see, e.g., [20]), and the idea of employing such covers, exciting higher-order polariton resonances, in order to synthesize super-directive radiators has been presented in [21]. As it happens in other applications [13]-[15], in these antenna problems the induced resonance happens fundamentally at the interface between materials with oppositely-signed constitutive parameters, allowing to squeeze the resonant dimensions of these devices just around these "complementary" interfaces [22], which do not depend any more on their total electric size, but rather on specific filling ratios between the metamaterials mentioned above and materials with positive constitutive parameters [which, for consistency, we name double-positive (DPS)].

Applying these concepts to patch antennas is relatively straightforward. A patch antenna resonance is closely associated with the cavity resonance of the volume between the patch and the ground plane, closed at its sides by magnetic walls [23], [24]. Mahmoud, inspired by the idea presented in [13], has recently proposed a novel layout for squeezing the resonant dimensions of circular ring patch antennas by loading it with a concentric pair of DPS and DNG materials [25]. Applying a cavity model to his geometry, in fact, he has shown that a proper pairing of DPS and DNG materials may lead in principle to a 'quasi-static' resonant frequency, independent of the metallic circular ring physical dimensions, but only depending on the filling ratio of the substrate underneath the patch. Even though dispersion and material loss were not considered in [25], and the antenna radiating features in this subwavelength case have not been analyzed, this interesting theoretical result has motivated several groups to investigate the topic more thoroughly. The results of some related studies have been presented in a recent conference [26]-[28]. Ermutlu and Tretyakov [26] have considered the subwavelength resonances of a rectangular patch antenna loaded with a DPS-DNG pair, considering also the DNG material dispersion (which is necessary and non-negligible in any passive material at the frequencies characterized by negative parameters [29]). They showed how the DPS-DNG loading may reduce considerably the resonant frequency also in this rectangular geometry [26] for the dominant mode. Petko and Werner have shown how it is possible to excite the $n=0$ (i.e., azimuthally independent) resonant mode in subwavelength circular patches [27]. All these results [25]-[27] mentioned above, however, have considered only the possibility that resonant modes may be supported by such subwavelength

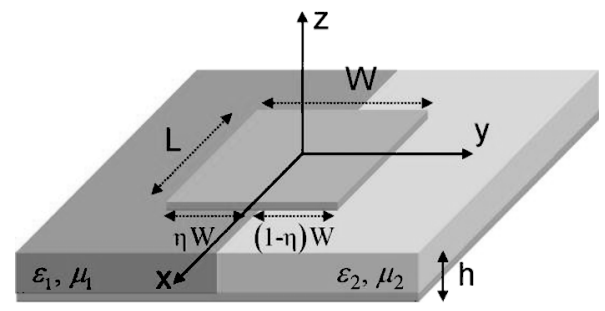

Fig. 1. A rectangular patch antenna loaded with a transversally inhomogeneous substrate.

radiators, without considering their radiation properties. As we show in the following, in fact, these proposed layouts may not properly radiate, unless specific modes are selected and excited. Portions of these results, together with some of the analyses reported in the following, have been orally presented by the authors in [28].

In this paper, we thoroughly revisit the theory of patch antennas loaded by metamaterials to show how a suitable pairing of metamaterials and standard dielectrics may indeed allow a subwavelength resonance in such structures. We find the necessary and sufficient conditions to get such quasi-static resonances for patch antenna setups. Then, we study theoretically the radiation properties of such subwavelength patches, showing which configurations may be designed to properly radiate in free space. Finally, we verify some optimized designs through full-wave numerical simulations, taking into account dispersion, losses and feeding networks for these devices. The results we present are encouraging for a future practical realization and may represent an interesting alternative way to build compact and efficient patch radiators.

\section{CAVITy Model: Resonant FreQuencies AND RADIATION PROPERTIES}

\section{A. Rectangular Patch}

Consider the rectangular patch antenna depicted in Fig. 1. It consists of a metallic patch with transverse dimensions $L \times W$ placed over a ground plane (distant $h$ ). The underneath substrate is inhomogeneous, filled with two isotropic and homogeneous materials with permittivity and permeability $\varepsilon_{1}(\omega), \mu_{1}(\omega)$ and $\varepsilon_{2}(\omega), \mu_{2}(\omega)$, in general varying with frequency (an $e^{j \omega t}$ notation is adopted in the following). The quantity $\eta$ represents the filling ratio of the volume underneath the patch, as described in the figure. The antenna is embedded in a suitable Cartesian reference system, as defined in the figure, and it radiates in free space, with permittivity and permeability $\varepsilon_{0}, \mu_{0}$.

The resonant frequencies of the radiator in Fig. 1 may be evaluated with good approximation by applying a standard cavity model [23], [24]. The resonant frequencies of the equivalent cavity for the $T M_{0 m 0}$ modes may be easily obtained by applying all the boundary conditions, and they correspond to the solution of the following dispersion equation:

$$
\frac{k_{1}}{\omega \mu_{1}} \tan \left[k_{1} \eta W\right]=-\frac{\omega \varepsilon_{2}}{k_{2}} \tan \left[k_{2}(1-\eta) W\right]
$$

where $k_{i}=\omega \sqrt{\varepsilon_{i} \mu_{i}}$ with $i=0,1,2$. 
Substituting the tangent functions with their arguments under the assumption of subwavelength size of the patch, i.e., $W \ll$ $\min \left[2 \pi / k_{1}, 2 \pi / k_{2}\right]$, yields the approximate equation

$$
\frac{\eta}{1-\eta} \simeq-\frac{\varepsilon_{2}}{\varepsilon_{1}}
$$

If one hypothetically assumes that $\varepsilon_{1}$ and $\varepsilon_{2}$ are independent of frequency (which they are not, [29]), this expression is surprisingly independent of $\omega$ and $W$, implying that in the first approximation the patch of Fig. 1 may resonate at any arbitrarily low frequency for any arbitrarily small width of the patch, provided that the previous equality among the filling ratio $\eta$ and the permittivities of the two materials is satisfied. (In other words, for specific values of the permittivities filling this equivalent cavity, in principle there is not a lower frequency limit below which a cavity of given total dimensions cannot resonate. This clearly overcomes, at least in principle, any other classical technique for squeezing the dimensions of such class of antennas.) Not surprisingly however, this inequality implies that the two permittivities are oppositely signed in the two materials, since $0<\eta<1$ makes the left-hand side of (2) a strictly positive quantity. Clearly, in fact, such a patch cannot resonate at low frequency unless special metamaterials are employed. The fact that an ENG, MNG or DNG material is necessarily dispersive with frequency [29] ensures the indirect dependence of the previous dispersion relation on frequency. (Also, from a practical point of view, constructing metamaterials at a very low frequency is a challenging task, if a highly subwavelength resonant inclusion is desired. Therefore this issue introduces at some point a practical lower limit for the size of the patch.) These findings, consistent with the subwavelength cavities, waveguides, scatterers and antennas described in [13]-[17], for which again a filling ratio factor, rather than their total size, determines the resonance of the system, may be justified by noticing that at the interface between materials with oppositely signed permittivities and/or permeabilities a local plasmonic resonance arises, which, if properly designed, may induce a resonance in the whole component. Notice how, due to the polarization of the mode under analysis $\left(T M_{0 m 0}\right.$, with electric field $E_{z}$ and magnetic field $H_{x}$ tangential to the interface) and due to the magnetic boundary conditions at the side walls of the cavity, in the quasi-static limit in which (2) applies the permittivities play a dominant role in the possibility of achieving such quasi-static resonance. The possibility of this resonant behavior in subwavelength rectangular patch antennas has been predicted applying a different approximate technique (i.e., the transmission line model) in [26].

Applying the dispersion relation given by (1), it is possible to determine the resonant frequencies of a given patch varying its medium loading. Fig. 2, as an example, shows the resonant frequency variation, as solution of (1), for a patch of width $W=50 \mathrm{~mm}$ with $\eta=0.5$ having one of the two media being a DPS material with $\varepsilon_{1}=2 \varepsilon_{0}, \mu_{1}=\mu_{0}$ and varying the permittivity of the other material $\varepsilon_{2}$. In particular, Fig. 2(a) shows the variation of the resonant frequency with $\varepsilon_{2}$ assuming $\mu_{2}=\mu_{0}$, whereas Fig. 2(b) shows the resonance frequency variation versus the plasma frequency $\omega_{e p}$ assuming a Drude dispersive material for the permittivity of the loading ENG material,

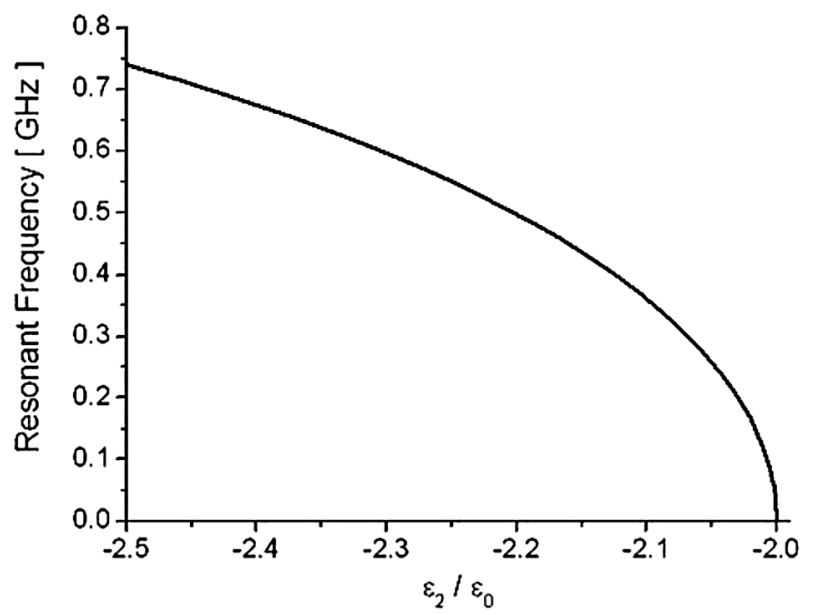

a)

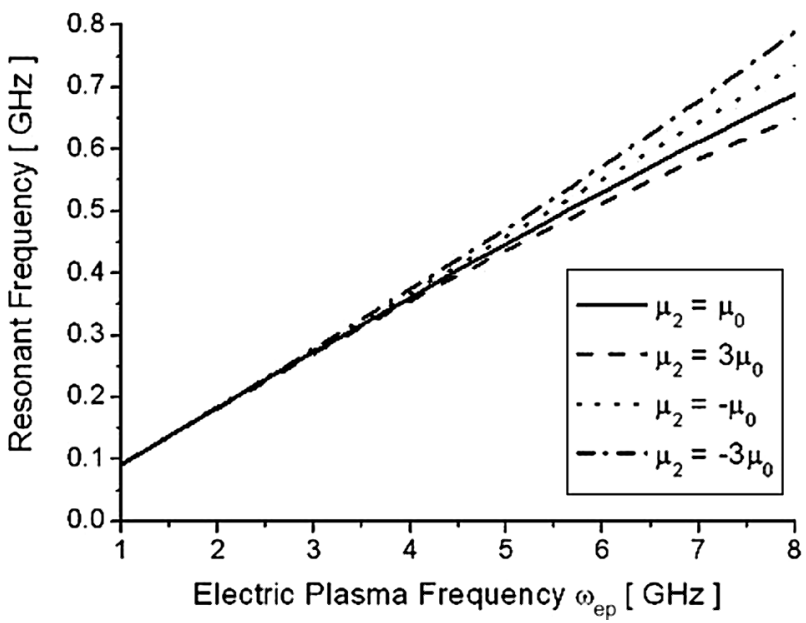

Fig. 2. (a) Variation of the resonant frequency of the rectangular patch antenna of Fig. 1 with $W=50 \mathrm{~mm}, \eta=0.5, \varepsilon_{1}=2 \varepsilon_{0}, \mu_{1}=\mu_{2}=\mu_{0}$ as a function of $\varepsilon_{2}$. (b) the same as a function of the electric plasma frequency of a Drude-like dispersive metamaterial for the second medium, varying also its permeability $\mu_{2}$ as a parameter.

i.e., $\varepsilon_{2}(\omega)=\varepsilon_{0}\left(1-\omega_{e p}^{2} / \omega^{2}\right)$. In this second case, $\mu_{2}$ is also varied as a parameter.

When the filling material is homogeneous, i.e., $\varepsilon_{1}=\varepsilon_{2}=$ $2 \varepsilon_{0}$, the patch has its resonance at $f=\omega / 2 \pi=2.12 \mathrm{GHz}$. However, loading the patch with an ENG material can reduce the resonance frequency in principle without limits, as shown in Fig. 2(a). When the permittivity $\varepsilon_{2}$ approaches the limit predicted by, which for the case at hand is $\varepsilon_{2}=-2 \varepsilon_{0}$, the resonance frequency may be made arbitrarily low (here material losses are being neglected, which would eventually limit this possibility at some point). Fig. 2(b) relates this variation to the plasma frequency of a Drude-like dispersive material. This has been adopted to model the realistic dispersion of an ENG metamaterial made by embedding specific inclusions inside a host material (at microwaves $\epsilon$-negative materials may be synthesized by including conducting wires with specific size, length and density in a host material). The value of the plasma frequency may be directly related to the geometric properties of such inclusions designed to synthesize the ENG material (see, for instance, [30] and [31]). Also in this case, properly varying the size and shape of the inclusions that form the second filling material (and consequently its electric plasma frequency $\omega_{e p}$ ) 
in order to approach the effective permittivity of $\varepsilon_{2}=-2 \varepsilon_{0}$, the resonance frequency may in principle be brought down arbitrarily. These results, as Fig. 2(b) shows, are weakly affected by the permeability of the second material $\mu_{2}$, and this dependence becomes irrelevant when the resonant frequency is reduced, since the quasi-static approximation represented by (2) holds. It is interesting to notice that in order to bring the resonant frequency down for a fixed patch dimension, it is known that a filling material with high electric permittivity may be employed instead of such plasmonic metamaterial. This solution, however, is intrinsically limited by the fact that a higher permittivity usually accompanies higher losses and the presence of surface waves, and reducing the resonance frequency to very low values would require the use of extremely high permittivities (for a fixed limited value of $e$, moreover, there is still a limit in the size of the patch to hold a resonance, whereas in this case there is in principle no limit to this size). On the other hand, the resonance is not obtained by adjusting the wavelength in the filling material, but instead by inducing a plasmonic resonance at the interface underneath the patch, which allows a phase cancellation similar to the effect predicted in [13].

This point is further clarified in Fig. 3, which shows the electric and magnetic field distributions at resonance predicted by the cavity model for the patch considered in Fig. 2 for the three cases when: (solid line) $\varepsilon_{2}=2 \varepsilon_{0}$, i.e., when the substrate is homogeneous and the patch resonates at $f=2.12 \mathrm{GHz}$; (dashed line) for a second material chosen according to (1) to lower the patch resonance ${ }^{1}$ to $f=0.5 \mathrm{GHz}$, i.e., using a material with $\varepsilon_{2}(f=0.5 \mathrm{GHz})=-2.2 \varepsilon_{0}$; (dotted line) for a second material with its permittivity increased so that the patch can resonate at the frequency $f=0.5 \mathrm{GHz}$, i.e., with $\varepsilon_{2}=140 \varepsilon_{0}$. The magnetic field amplitudes have been normalized in the figure to their relative maximum and the electric field are normalized accordingly for comparison. As it can be seen, the three cases show very different properties. The standard resonance of the patch would happen at $f=2.12 \mathrm{GHz}$, as the solid line shows. At this frequency the patch width is $\lambda / 2$, with $\lambda$ being the wavelength inside the homogeneous material loading the patch. As it is well known, the magnetic field in fact experiences a half-wavelength sinusoidal variation from one side to the other of the patch, whereas the electric field flips its sign, with a $180^{\circ}$ phase variation. Filling the region $y>0$ with a material with $\varepsilon_{2}(f=0.5 \mathrm{GHz})=-2.2 \varepsilon_{0}$ allows getting a resonance at $f=0.5 \mathrm{GHz}$, as shown by the dashed line. In this case the magnetic field flips the sign of its derivative, due to the boundary conditions at the interface between the two "oppositely signed" materials, and this allows to shrink the electrical dimensions of the equivalent cavity, similarly to what was speculated in [13], i.e., exploiting the phase-compensating properties of negative materials. As clearly seen in the figure, the electric field variation in this case is almost constant and its phase does not flip

\footnotetext{
${ }^{1}$ It should be emphasized again that the choice of a resonance at $f=0.5 \mathrm{GHz}$ in this, and the next examples, is arbitrary since as Fig. 2 shows, in principle the patch may resonate even at lower frequencies. In this case, its dispersion relation (2), in fact, depends on the filling ratio of the equivalent cavity closed between the patch and the ground plane, rather than on its total dimension, and this is the main point behind the present technique of using metamaterials to reduce the patch antenna dimensions. This choice, therefore, which was made for the numerical examples here, does not represent a lower limit in this case.
}
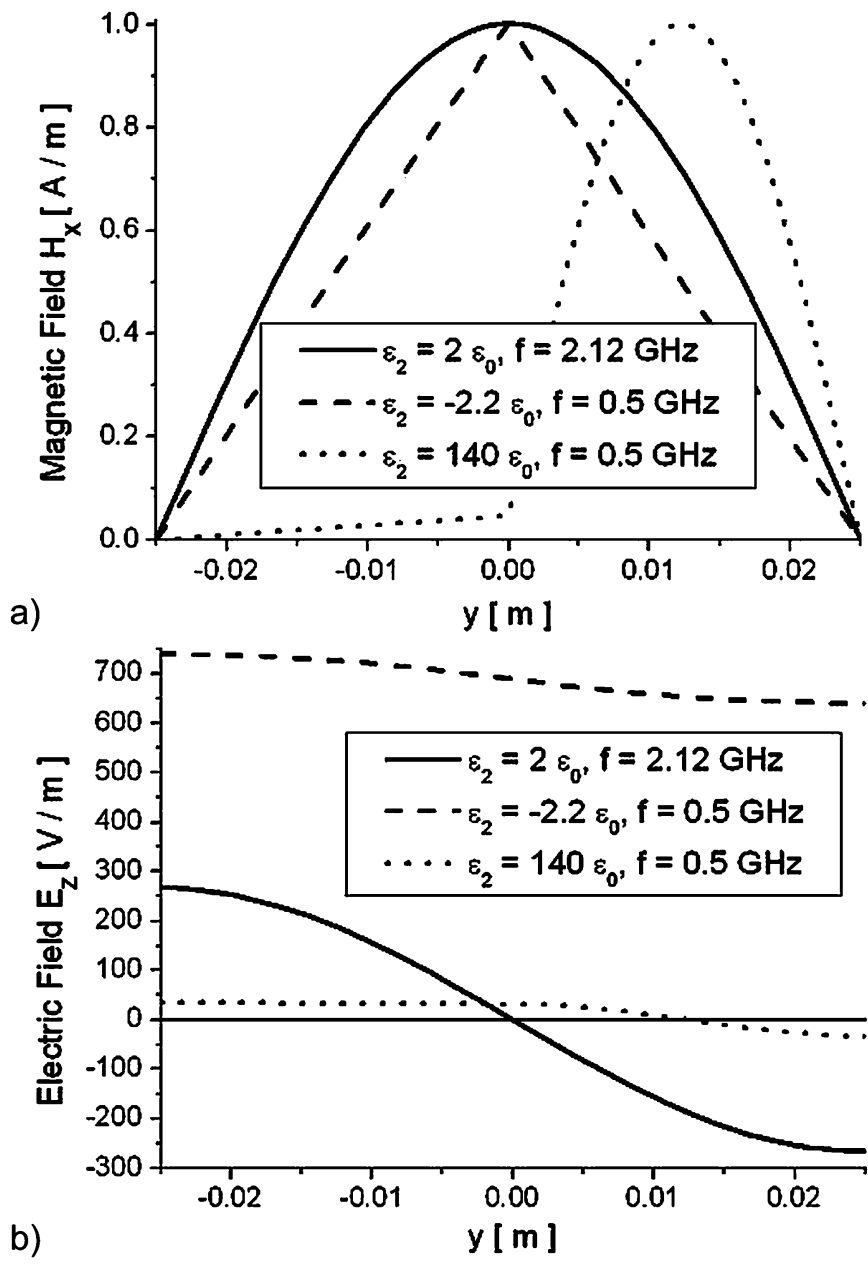

Fig. 3. Variation of the (a) magnetic and (b) electric fields (as predicted by the cavity model) underneath the rectangular patch antenna of Fig. 2, at resonance, with: $\varepsilon_{2}=2 \varepsilon_{0}$ (solid line), resonating at $f=2.12 \mathrm{GHz} ; \varepsilon_{2}=-2.2 \varepsilon_{0}$ (dashed line), resonating at $f=0.5 \mathrm{GHz} ; \varepsilon_{2}=140 \varepsilon_{0}$ (dotted line), resonating at $f=0.5 \mathrm{GHz}$.

passing from one side to the other of the patch. This affects the radiation properties of the patch, as we will show in the following. Employing a high-dielectric material (dotted line), the resonance frequency is made low by decreasing the wavelength in the material in $y>0$, which in fact is responsible for almost all the sinusoidal variation of the magnetic field. The electric field remains almost constant in the left material, but experiences a $180^{\circ}$ phase shift in the material with high permittivity.

Even though the three cases depicted in Fig. 3 all correspond to resonances underneath the patch, their radiation features are quite different. The radiation properties can be easily predicted in the first approximation by applying an effective aperture model [1], [2]. The well-known cause of radiation is represented by the fringing electric field at the two sides of the patch, i.e., at $y= \pm W / 2$. They correspond to the equivalent magnetic currents $\mathbf{K}=-\hat{\mathbf{n}} \times\left.\mathbf{E}\right|_{y= \pm W / 2}$, where $\mathbf{E}$ is the electric field at $y= \pm W / 2$, as predicted by the cavity model, and $\hat{\mathbf{n}}$ is the normal to the side of the patch, i.e., $\hat{\mathbf{n}}= \pm \hat{\mathbf{x}}$, respectively. In the solid and dotted line cases, i.e., when the cavity is resonating in a conventional way, the electric field on the two sides of the patch is oppositely oriented, and therefore 
the two sides of the patch radiate in phase towards broadside. This ensures the conventionally shaped radiation pattern of the patch antenna, with reasonable directivity at broadside. In the dot-line case, when a high permittivity is employed to squeeze the patch width, we may expect a lower directivity, due to the reduced electrical distance between the two radiating edges, and a lowered gain caused by the presumably strong excitation of surface waves, due to the presence of a high- $\epsilon$ substrate. Also the potential presence of high losses in such material would contribute to deterioration of its radiating performance. When the patch is loaded with a metamaterial, however, the mode excited (dashed line) will cause the two sides of the patch to radiate out of phase. The radiation pattern, therefore, is expected to have a null in the broadside direction. Due to the electrically small dimensions of the patch, moreover, the two magnetic currents almost cancel each other for all the visible angles, and thus the radiation efficiency of such an antenna would be very poor. In other words, such a subwavelength rectangular patch may act more as a resonator rather than as an antenna and the ratio of stored versus radiated energy is expected to be extremely high for electrically small patches. Another aspect should also be considered: the interfaces at $y=0$ and $\left\{\begin{array}{l}z=0 \\ y>0\end{array}\right.$ support surface plasmons, since they are interfaces between media with materials with opposite permittivities [32]. If the plasmonic resonance at $y=0$ is the main factor responsible for the subwavelength resonance, these surface plasmons, when excited, would eventually trap some energy from the source, thus further reducing the radiation efficiency of the antenna.

Following the previous considerations, it is clear that the rectangular geometry described in Fig. 1 is not a valid option for the purpose of designing an efficient subwavelength radiator. We show in the following that the circular geometry may provide additional degrees of freedom for selecting the proper mode of operation in order to get an efficient radiation from a subwavelength radiator loaded with metamaterials.

\section{B. Circular Patch}

The main reason why the rectangular patch of Fig. 1 could not provide an efficient radiation is represented by the impossibility of selecting a higher-order resonant mode in the equivalent cavity formed by the subwavelength patch loaded with metamaterials. Only the fundamental mode can be excited in such a subwavelength rectangular cavity, providing, thus, out-of-phase radiation from the radiating edges of the patch. Matching features may in principle show a subwavelength resonance tuned at the desired frequency, while the gain of the radiator is expected to be very poor. The parallel plate cavity studied in [13] showed analogous limitations: there are no degrees of freedom in selecting the desired operating mode in its subwavelength regime of operation. However, in [15] it was shown how it is possible to select the desired resonant mode in subwavelength cylindrical and spherical scatterers, acting as open subwavelength resonators. Following a similar argument, we consider here the circular patch geometry to overcome the problems highlighted in the previous section.

The circular patch under analysis is depicted in Fig. 4. It is loaded by a grounded inhomogeneous substrate, with thickness

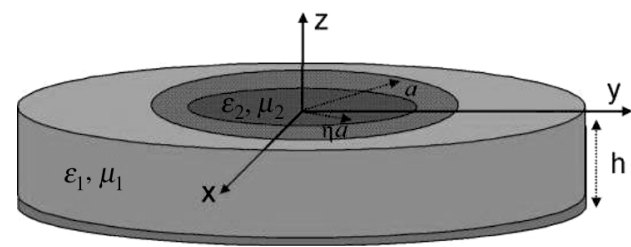

Fig. 4. A circular patch antenna loaded with a radially inhomogeneous substrate.

$h$, consisting of a planar layer with permittivity and permeability $\varepsilon_{1}(\omega), \mu_{1}(\omega)$ with a core ring with permittivity and permeability $\varepsilon_{2}(\omega), \mu_{2}(\omega)$. The patch radius is $a$ and the core radius is $\eta a$, with $0<\eta<1$. The quantity $\eta$ again represents the filling ratio of the volume underneath the patch.

A cavity model may again be applied to this problem, looking for the resonant frequencies of the cavity which is obtained by closing the volume underneath the patch with a cylindrical magnetic wall. Matching the boundary conditions provides the following dispersion relations for the $T M_{m n 0}$ modes:

$$
\mu_{2} \frac{J_{n}\left(k_{2} \eta a\right)}{J_{n}^{\prime}\left(k_{2} \eta a\right)}=\mu_{1} \frac{J_{n}\left(k_{1} \eta a\right) Y_{n}^{\prime}\left(k_{1} a\right)-Y_{n}\left(k_{1} \eta a\right) J_{n}^{\prime}\left(k_{1} a\right)}{J_{n}^{\prime}\left(k_{1} \eta a\right) Y_{n}^{\prime}\left(k_{1} a\right)-Y_{n}^{\prime}\left(k_{1} \eta a\right) J_{n}^{\prime}\left(k_{1} a\right)}
$$

where $J_{n}($.$) and Y_{n}($.$) represent, respectively, the Bessel and$ Neumann cylindrical Bessel functions, as commonly defined (see, e.g., [33]) and $n$ is the angular order of the mode $\left(e^{-j n \varphi}\right.$ variation, $\varphi$ being the azimuthal angle in cylindrical coordinates). Again, under the same quasi-static assumptions applied to the rectangular case, which is $a \ll \min \left[2 \pi / k_{1}, 2 \pi / k_{2}\right]$, (3) becomes

$$
\begin{gathered}
\frac{\eta^{2}}{1-\eta^{2}} \simeq-\frac{\varepsilon_{1}}{\varepsilon_{2}} \quad n=0 \\
\frac{1-\eta^{2 n}}{1+\eta^{2 n}} \simeq-\frac{\mu_{1}}{\mu_{2}} \quad n>0
\end{gathered}
$$

which is the analogous of (2), but in the circular geometry. We also note here that the dispersion relation is a function of the filling ratio $\eta$ and of the constitutive parameters of the two materials and that the relations in (4) again imply the use of oppositely-signed materials. However, this time the dispersion relations vary as a function of the angular order of the resonant mode. This interesting peculiarity allows tuning the filling ratio and/or the material parameters in order to select the proper angular variation for the mode, which may also allow selection of the desired radiation properties of the subwavelength patch, as anticipated earlier. This is in analogy with what was found in cylindrical subwavelength scatterers at resonance in [15] (notice also the analogy in the dispersion formulas to get a subwavelength resonance). It is interesting to note that a circular patch loaded with regular DPS materials has a dominant $T M_{110}$ mode, as easily shown in the homogeneous case [1]. Here, however, by employing oppositely-signed materials it is possible in principle to choose the desired angular variation for the dominant mode of the subwavelength cavity and tailor its resonance at the desired frequency. 


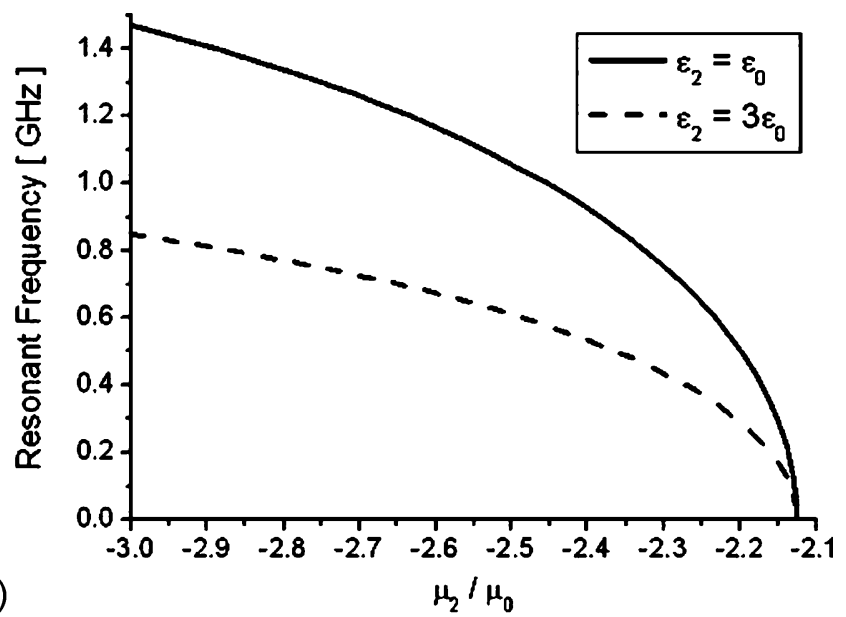

a)

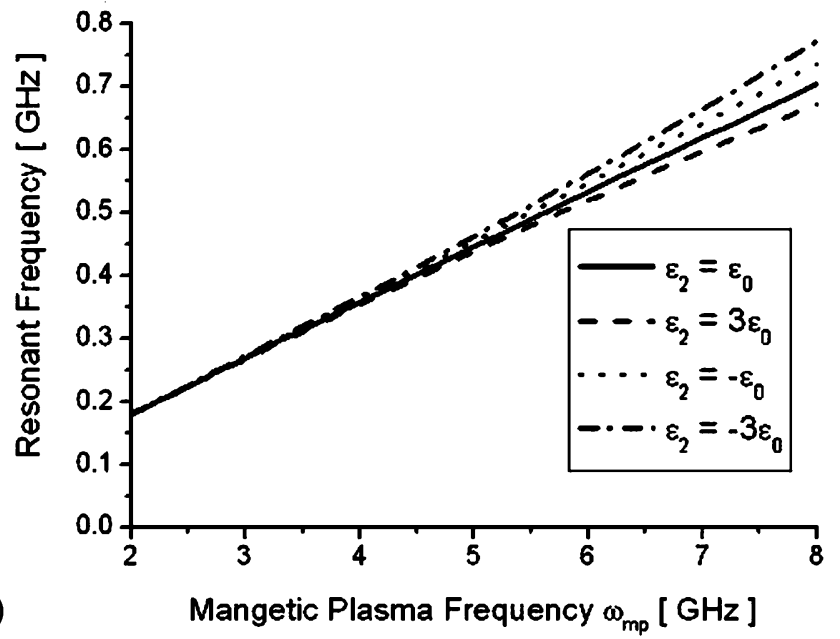

Fig. 5. (a) Variation of the resonance frequency of the circular patch antenna of Fig. 4 with $a=20 \mathrm{~mm}, \eta=0.6, \varepsilon_{1}=3 \varepsilon_{0}, \mu_{1}=\mu_{0}$ as a function of $\mu_{2}$ with $\varepsilon_{2}$ as a parameter for the $T M_{110}$ mode of operation. (b) The same as a function of the magnetic plasma frequency of a Drude-like dispersive metamaterial for the second medium. Here $\varepsilon_{2}$ is varied as a parameter.

Fig. 5, analogous to Fig. 2 but for this circular geometry, provides the variation of the resonance frequency in terms of the permeability of the inner core material for an example of circular patch with $a=20 \mathrm{~mm}, \eta=0.6, \varepsilon_{1}=3 \varepsilon_{0}, \mu_{1}=\mu_{0}$. In this case the mode $T M_{110}$ is considered, which is, as we show below, the only one giving rise to effective radiation. Due to the different boundary conditions in the cavity, now the permeabilities play a dominant role for this mode in the quasi-static limit, and this is why a MNG material is required to yield a subwavelength resonance for this mode (the $n=0$ mode, as indicated by (4), is the only one in which the permittivities dominate, analogously to what happens for the cylindrical scatterer problem [15]). Again, it is evident how it is possible to reduce considerably the resonance frequency of the patch (which in this case would be at $f=2.54 \mathrm{GHz}$ if a homogeneous substrate with $\varepsilon_{1}=\varepsilon_{2}=3 \varepsilon_{0}$ were considered) by varying the core permeability. Fig. 5(b), similar to Fig. 2(b) for the rectangular case, considers as an example a Drude dispersion for the core permeability as $\mu_{2}(\omega)=\mu_{0}\left(1-\omega_{m p}^{2} / \omega^{2}\right)$ and has $\varepsilon_{2}$ as a parameter. Metamaterials with resonant permeabilities (and therefore negative permeability in a given frequency range) may be synthesized at microwave frequencies by embedding splint ring res- onators (SRR) or similar loop inclusions in a host dielectric, as experimentally shown in the recent years by several groups (e.g., [34] and [35]). ${ }^{2}$

The field variation for the different modes at resonance, as predicted by the cavity model, is shown in Fig. 6 for the circular patch with geometry described in the caption of Fig. 5 as a function of the radial coordinate $\rho$ (here the tangential fields with respect to the interface, i.e., $E_{z}$ and $H_{\varphi}$ are shown; for modes with $n \geq 1$ the normal component $H_{\rho}$ would also be present, and is proportional to $\mu H_{\varphi}^{\prime}$ ). Fig. 6(a) refers to the mode with no angular variation, i.e., $n=0$. Its resonance for a homogeneous substrate (i.e., with $\varepsilon_{2}=3 \varepsilon_{0}, \mu_{2}=\mu_{0}$ ) would happen at $f=5.28 \mathrm{GHz}$ (solid line), but it can be brought down to $f=0.5 \mathrm{GHz}$ by employing an ENG material with $\varepsilon_{2}=-5.44 \varepsilon_{0}$ (dashed line), or by increasing the permittivity of the second material to the enormous value of $\varepsilon_{2}=925 \varepsilon_{0}$. In the dashed line case this mode would become the dominant mode of the patch, and the typical V-shaped distribution of the phase compensation of negative materials is evident from the magnetic field distribution. Notice that the dotted line solution would be characterized by other resonant modes with lower frequency, since the extremely high permittivity of the second material implies that this patch is not subwavelength at all (in this case in fact a $n=1$ mode would be dominant, resonating at $f=0.28 \mathrm{GHz}$ ). This mode of operation, when the ENG material is employed (dashed line), is in many senses analogous to the rectangular cavity previously analyzed. Indeed the electric field remains somewhat constant in the cavity and does not flip its sign from the center to the side of the patch, as it usually does in the regular case.

In order to excite a different mode, we can play with (4) and select a proper MNG material to yield the required resonance of higher- $n$ modes. For instance, the mode $n=1$ may be excited at the desired $f=0.5 \mathrm{GHz}$ by choosing $\mu_{2}=-2.36 \mu_{0}$, as shown by the dashed line (here, the permittivity was kept fixed at $\varepsilon_{2}=$ $3 \varepsilon_{0}$ ) in Fig. 6(b). Consistent with the previous findings, here also a flip in sign of the derivative of the tangential magnetic field allows shrinking the electrical dimensions of the patch. The use of a high-permeability material (dotted line) might lead again to high losses and difficulties in realizing such very high values of permeability, (and in this case also very high electric field with related matching problems). Also the mode $n=2$ may become dominant using a different MNG core, as shown in Fig. 6(c).

It is interesting to point out that, different from the rectangular case, in this geometry the dependence of the field along the side wall of the cavity $\rho=a$, which, as we have seen, is directly related to the radiating features of the patch, is not affected by the subwavelength size of the patch, but it is uniquely determined by the choice of the order $n$ of angular variation of the mode. This implies that a proper choice of this variation in the subwavelength case may lead to a subwavelength patch with efficient radiation properties.

\footnotetext{
${ }^{2}$ The frequency dispersion of magnetic metamaterials synthesized using SRR inclusions is generally modeled with a Drude-Lorentz generalized model. For sake of simplicity here we have assumed a simple Drude model, which also ensures causality, satisfies Kramers-Kronig relations, and approximates more complex frequency dispersions over limited frequency ranges. These and the following results may be easily verified for more relevant Lorentz dispersion models.
} 

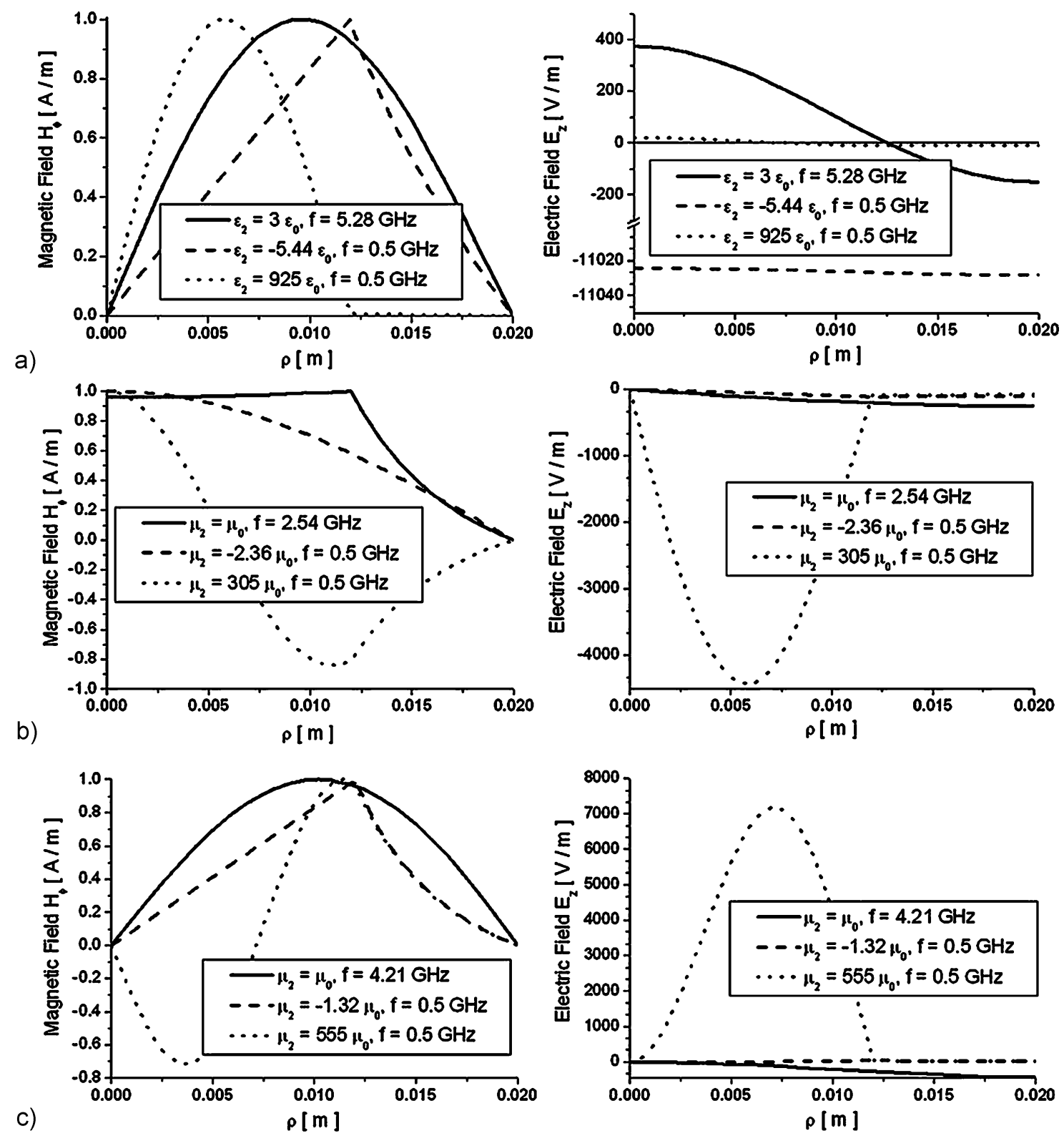

Fig. 6. Variation of the magnetic (left column) and electric (right column) fields (as predicted by the cavity model) underneath the circular patch antenna of Fig. 5, at resonance, for the different modes (a) $n=0$, (b) $n=1$, (c) $n=2$.

As in the rectangular case, the radiation properties of the antenna of Fig. 4 are indeed directly related to the equivalent magnetic currents radiating on the patch side at $\rho=a$ and determined by the fringing electric field as $\mathbf{K}=-\hat{\boldsymbol{\rho}} \times\left.\mathbf{E}\right|_{\rho=a}$. The total power radiated by these currents in the spherical direction $(\theta, \varphi)$ is known to be proportional to the following integral [1], [2]:

$$
P_{\text {rad }} \propto \int_{0}^{2 \pi} e^{-j n \phi^{\prime}} \sin \left[n\left(\phi-\phi^{\prime}\right)\right] e^{j k_{0} a \sin \theta \cos \left(\phi-\phi^{\prime}\right)} d \phi^{\prime}
$$

which in the quasi-static limit of $k_{0} a \ll 1$ reduces to

$$
P_{\text {rad }} \propto \int_{0}^{2 \pi} e^{-j n \phi^{\prime}} \sin \left[n\left(\phi-\phi^{\prime}\right)\right] d \phi^{\prime}=j \pi e^{-j \phi} \delta(n-1)
$$

where $\delta($.$) is the Kronecker delta function, being unity when$ its argument is zero and being zero otherwise. As easily confirmed by inspecting the electric field orientation for the different modes in Fig. 6. Equation (6) states that the mode $T M_{110}$ would produce a non-zero radiated field even in the limit of vanishing electrical dimensions of the patch, due to the correct phase variation around the patch side. Its dependence on $\theta$ and therefore its directivity is reduced, due to the small electrical dimensions of the magnetic current loop, but the fact that it is radiating efficiently is not affected when the patch electrical size is scaled down. All the other modal field configurations would share the same problem of radiation cancellation that we have verified in the rectangular case, even though the related resonances may happen at very low frequencies and the impedance matching may be acceptable at those specific frequencies.

At this point, it is worth noting that the other attempts recently presented in the literature by other groups [32]-[34] to design a 
subwavelength patch antenna with resonant features would face a similar radiation problem in a practical situation, since they did not consider the radiation properties of such antennas. The analysis presented in [25] for the subwavelength annular ring patch antenna, which analyzed the $T M_{110}$ mode applying the appropriate cavity model, has shown that this mode may be excited at a low-frequency resonance by properly loading the substrate with a DNG metamaterial, similar to the analysis shown here. The field distributions reported in [25], however, clearly show that in the limit of subwavelength size of the patch the two equivalent magnetic current loops at the outer and inner side of the ring would radiate out of phase with each other, producing a cancellation of the radiated field for a subwavelength patch. The possibility of exciting the $n=0$ mode in a subwavelength circular patch, as presented in [27], has also been shown to produce a poor radiator, as (6) testifies. The same may be said about the rectangular subwavelength patch analysis presented in [26], as we have previously shown. Other patch shapes, i.e., elliptical or more complex geometries, may be treated similarly to the analysis shown here, and proper resonant modes may be chosen to have more efficient radiation combined with a small size. This is not done here due to lack of space, but it may be the subject of a future publication.

In the following section, we verify these results with fullwave simulations conducted with the software tool (CST Microwave Studio [36]), considering the limitations that losses, small shape variations (due to the discretization that the numerical software applies) and the presence of the feed network may cause to the results shown here. We also calculate the relevant radiation properties in qualitatively realistic setups following these findings.

\section{NUMERICAL SIMULATIONS}

\section{A. Rectangular Patch}

As a first example we study the behavior of the rectangular patch antenna of Fig. 2, with $W=50 \mathrm{~mm}, L=40 \mathrm{~mm}$, $h=1.5 \mathrm{~mm}, \eta=0.5, \varepsilon_{1}=2 \varepsilon_{0}, \mu_{1}=\mu_{2}=\mu 0$, properly loaded with a Drude-dispersive and lossy ENG material with $\varepsilon_{2}(\omega)=\varepsilon_{0}\left(1-\omega_{e p}^{2} /\left[\omega\left(\omega-j \omega_{\tau}\right)\right]\right)$. The plasma frequency has been set at $\omega_{e p}=5.6 \mathrm{GHz}$ to get $\varepsilon_{2}(f=0.5 \mathrm{GHz}) \simeq$ $-2.2 \varepsilon_{0}$, which may be obtained in an artificial metamaterial by embedding properly designed conducting wires oriented along $z$ in vacuum or another dielectric [30], [31] (the polarization of the electric field underneath the patch is predicted by the cavity model, as described in the previous section). ${ }^{3}$ Losses in the metamaterial have also been added to reflect the possible ohmic losses in the conducting inclusions, and thus the damping frequency in the Drude model has been set at $\omega_{\tau}=50 \mathrm{MHz}$. The antenna is fed by a coaxial probe placed at the position $x_{p}=0, y_{p}=-W / 4$ with inner radius of $r_{i n}=0.3 \mathrm{~mm}$ and characteristic impedance $Z_{p}=125 \Omega$. Also finite substrate and ground pane have been considered, both with total size of $100 \times 100 \mathrm{~mm}^{2}$. In order to consider all the realistic aspects of the design, the finiteness of the metal thickness has also been

\footnotetext{
${ }^{3}$ Here we assume an isotropic medium. However, we claim that the required metamaterial does not have to be isotropic, due to the specific field polarization that is expected when properly exciting the resonant mode.
}
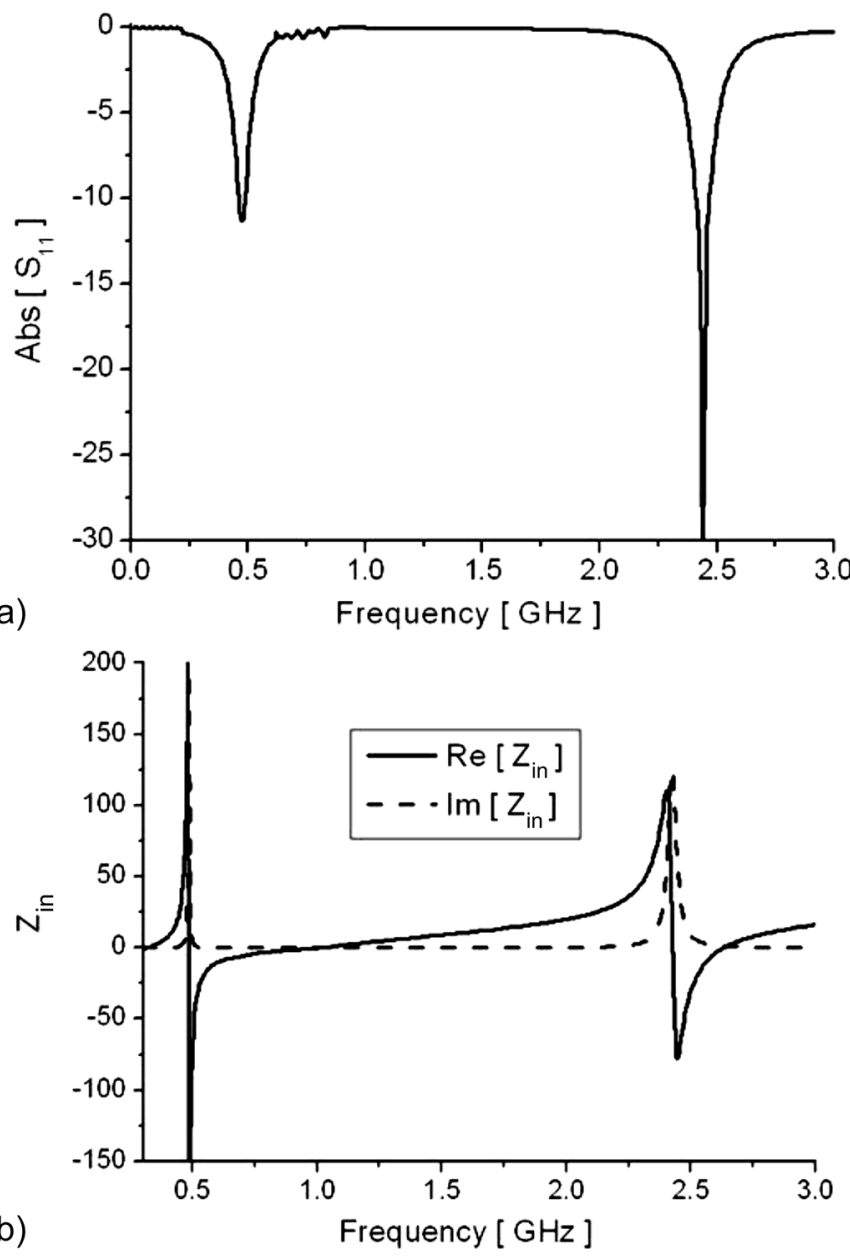

Fig. 7. Full-wave numerical simulation results for (a) return loss and (b) input impedance of the antenna in Fig. 2 with a Drude material with losses, fed by a coaxial cable, as described in the text.

modeled, fixing it at $0.01 \mathrm{~mm}$. The structure has been simulated with a finite-integration-technique commercial software (CST Microwave Studio [36]).

The return loss and the input impedance are reported in Fig. 7, showing two distinct resonances at $f=0.48 \mathrm{GHz}$ and at $f=$ $2.44 \mathrm{GHz}$, with good agreement with the cavity model. The first resonance is the subwavelength resonance described in the previous section, whereas the second one is a common resonance happening when the material permittivity of the metamaterial is not affected by the presence of the inclusions, since $\varepsilon_{2}(f=2.44 \mathrm{GHz}) \simeq \varepsilon_{0}$ and it may be predicted by using (1) with vacuum as the second material.

The subwavelength resonance in this case is poorly matched (however the position of the probe has not been properly optimized in this case, since the radiation properties at such frequency are expected to be poor anyhow, as shown in the previous section). The power taken from the feed is expected to be lost in ohmic losses or trapped into the plasmonic waves traveling along the interface $y=0$.

Fig. 8 reports the electric field distribution (a snapshot in time) for these two different resonance frequencies on the plane of the patch. This clearly shows how the fringing fields are oppositely directed in the subwavelength case, different from the usual resonance at $f=2.44 \mathrm{GHz}$. The field distributions are 
a)

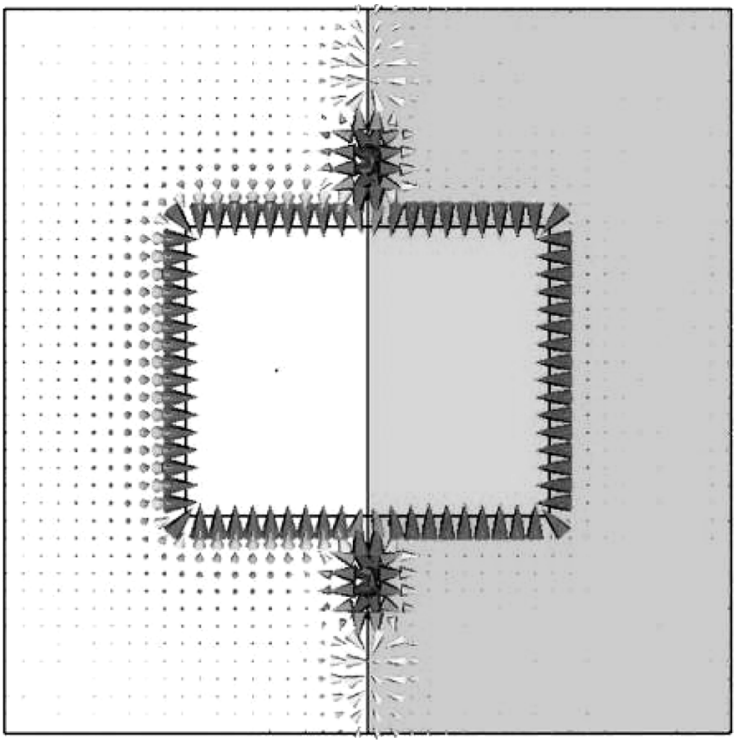

b)

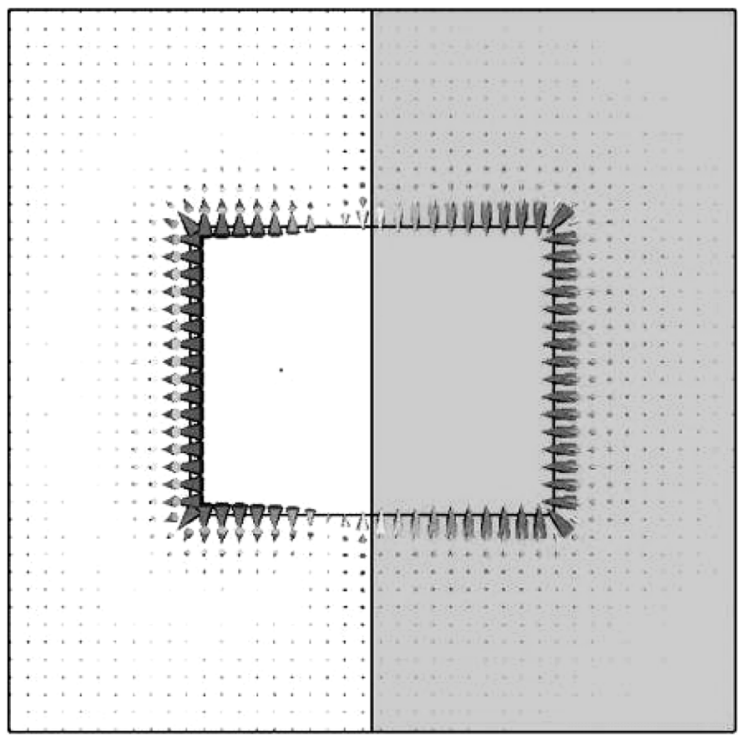

Fig. 8. (Results calculated using CST Microwave Studio [36]). Electric field distribution (snapshot in time, top view) at the first two resonant frequencies for the antenna of Fig. 7: at (a) $f=0.48 \mathrm{GHz}$ and (b) $f=2.44 \mathrm{GHz}$.

consistent with the cavity model analysis described in the previous section and confirm the different behavior of the field at the radiating edges at the two frequencies, highlighting also the standing waves present at the interface $y=0$ due to the excitation of surface plasmons. The radiation patterns in the two cases (not reported here) are substantially different, since at the lower frequency the patch is not radiating properly and it shows a null at broadside and an insufficient gain over all the visible angles. This rectangular configuration at the frequency $f=0.48 \mathrm{GHz}$ ends up being a good candidate for a matching network for the excitation of surface plasmons at the interface $y=0$ rather than as a good radiator.

These full-wave simulations confirm and validate numerically the results reported in the previous section, which were obtained with an approximate cavity model. As a next step we have designed and simulated a circular patch, following the guidelines of the previous analysis.
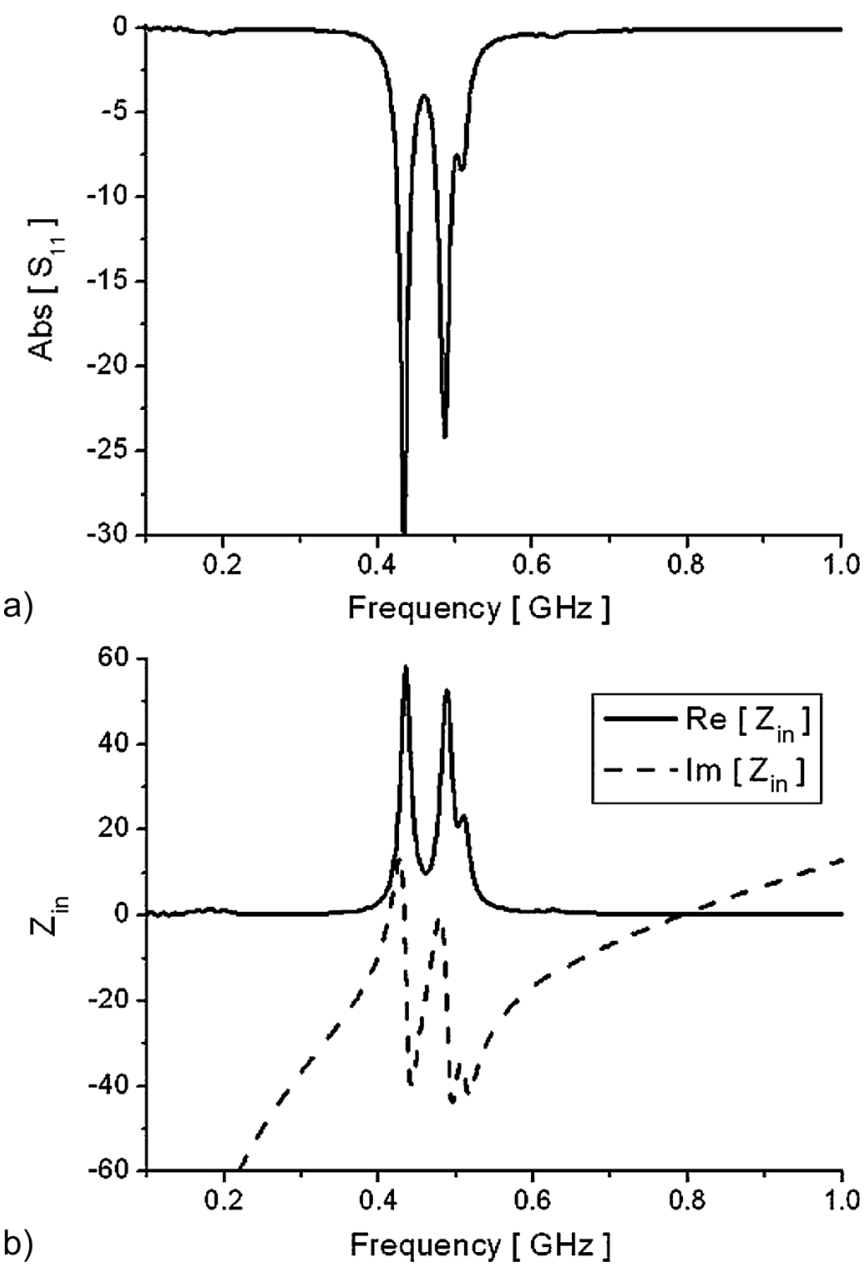

Fig. 9. Full-wave numerical simulation results for (a) return loss and (b) input impedance of the antenna in Fig. 5 with a Drude magnetic material with losses, fed by a coaxial cable, as described in the text.

\section{B. Circular Patch}

We have modeled the circular patch of Fig. 4, similar to the previous case, with the following parameters: $a=20 \mathrm{~mm}, \eta=$ $0.6, \varepsilon_{1}=\varepsilon_{2}=3 \varepsilon_{0}, \mu_{1}=\mu_{0}$. The outer dielectric slab and the ground plane were supposed to be finite with circular symmetry and outer radius $a=30 \mathrm{~mm}$. For a realistic implementation of the inner core with an MNG material, again a Drude model has been assumed with $\mu_{2}(\omega)=\mu_{0}\left(1-\omega_{m p}^{2} /\left[\omega\left(\omega-j \omega_{\tau}\right)\right]\right.$ and the thickness of the substrate has been increased to $h=$ $5 \mathrm{~mm}$ with respect to the previous design, in order to allow more spacing for future hosting the split-ring resonators to construct an MNG material (see, e.g., [34]). Similar results to those that are shown in the following have been obtained with thinner substrates any way, showing that this parameter does not affect sensibly the previous analysis, provided that it is maintained well below the other relevant dimensions of this geometry. The magnetic plasma frequency has been fixed at $\omega_{m p}=4.31 \mathrm{GHz}$. Applying (3), this should yield a resonance at $f=0.4 \mathrm{GHz}$. The damping frequency has been set at $\omega_{\tau}=50 \mathrm{MHz}$, again to take into account some possible ohmic losses in the conducting inclusions that will be needed for construction of the MNG metamaterial. The coaxial cable has been designed to have a $Z_{p}=50 \Omega$ characteristic impedance with $r_{i n}=0.2 \mathrm{~mm}$ and its position is at $\rho_{p}=0.75 a$. 


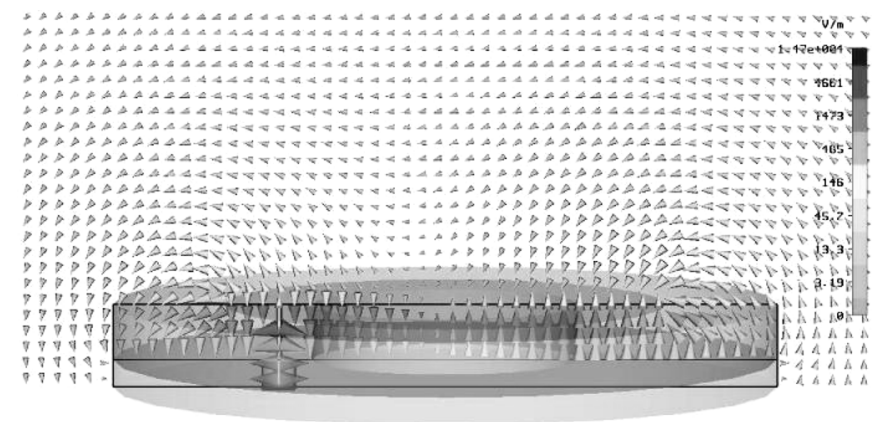

Fig. 10. (Results calculated using CST Microwave Studio [36]). Electric field distribution on the $y=0$ plane for the antenna of Fig. 9 at $f=0.47 \mathrm{GHz}$.

The matching properties of such configuration are reported in Fig. 9 and show that a good matching may be obtained over a certain frequency band. In this case, the full wave analysis shows a double resonance near the resonant properties, which may improve the bandwidth of operation. We underline that this is not an optimized setup, since our goal here is merely to verify the theory reported in the previous section. However, the results reported here are more than encouraging, and with optimized setups we expect to further improve this performance that may cause the two peaks to be closer and thus have a better bandwidth of operation. The agreement with the cavity model is again good and the input port of the antenna is matched using a simple coaxial cable with $50 \Omega$ impedance.

Fig. 10 shows the electric field distribution on the plane $y=0$ at the frequency $f=0.47 \mathrm{GHz}$, showing how, despite the small dimension of the patch, the two opposite sides of the patch can indeed radiate in phase. Although the patch is subwavelength, the excitation of the $n=1$ mode in fact allows the electric field to flip its sign passing from one side to the other side of the patch.

Another advantage of this configuration is that no surface plasmons carrying power away from the source are expected, due to the finiteness and "closedness" of the interface between MNG and DPS materials. The electric current induced on the metallic patch at $f=0.47 \mathrm{GHz}$ is depicted in Fig. 11. It clearly shows how the $n=1$ mode is at resonance, and it is indeed interesting to notice how the current can be "closed" in electrically small resonant loops, despite the small dimensions of the patch (its diameter at this frequency is just $6.5 \%$ of the free space wavelength).

The radiation pattern and gain for this configuration, as reported in Fig. 12, show indeed encouraging performance. The subwavelength patch radiates with a maximum gain at broadside of $1.8 \mathrm{dBi}$, testifying a reasonable efficiency and a good matching, which may offer interesting possibilities for designing subwavelength small radiators. You may notice how its directivity is less than the one obtained with a patch of standard dimension, due to the small electrical size of the effective radiating aperture, as expected from (6). Also, the front-to-back ratio is drastically lower than the one of a patch of regular dimensions, since the reduced size of the ground plane (its diameter here is only $9.4 \%$ of the wavelength) does not allow a significant reflection. We should emphasize, however, that our goal here was in fact to design a radiator, whose overall

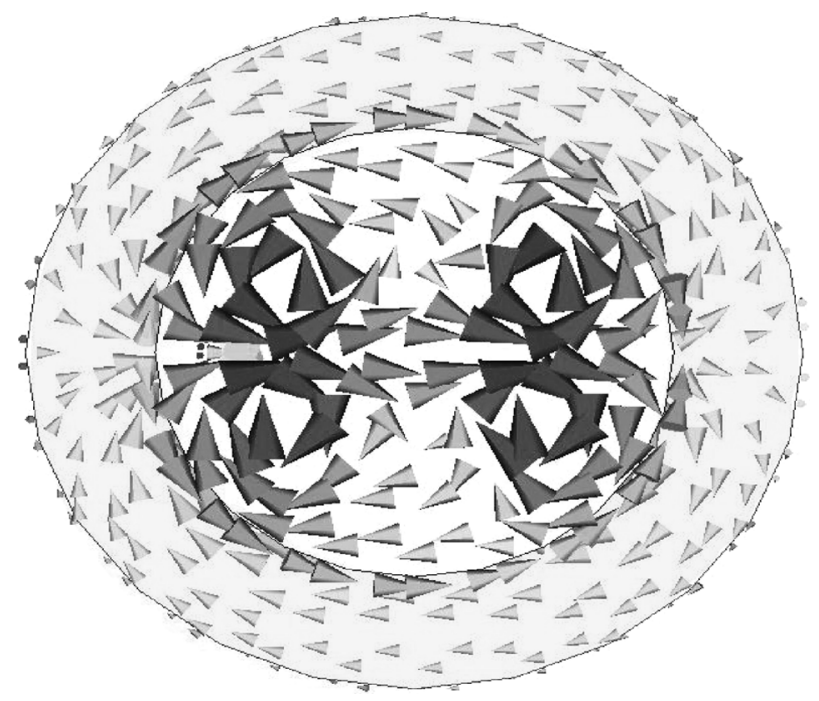

Fig. 11. (Results calculated using CST Microwave Studio [36]). Electric current distribution on the metallic patch and on the underlying ground plane for the antenna of Fig. 11 at $f=0.47 \mathrm{GHz}$.

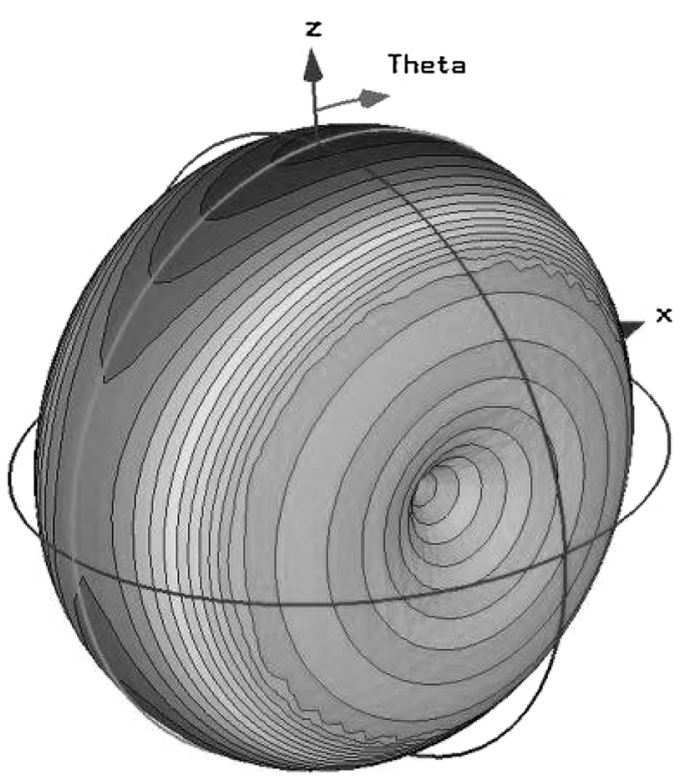

$\mathrm{dB} \mathbf{i}$

1.69

1.45

1.22

0.989

0.756

0.524

0.291

$-1.76$

$-4.11$

$-6.46$

$-8.8$

$-11.2$

$-13.5$

$-15.8$

$-18.2$

Fig. 12. (Results calculated using CST Microwave Studio [36]). Gain (radiation) pattern for the antenna of Fig. 11 at $f=0.47 \mathrm{GHz}$.

size is much smaller than the wavelength radiating efficiently in free space. The radiation of Fig. 12 consistently corresponds to the one of a short magnetic dipole radiating in free space, since the overall size of the system is much shorter than the operating wavelength. We have simulated the same antenna with a larger ground plane, and have obtained similar results but with an increased front-to-back ratio and therefore higher gain. These results are not reported here for sake of brevity.

To conclude, we briefly discuss here some practical issues concerning the realization of this miniaturized setup. It should be noted at first that the orientation of the magnetic field underneath the patch, well predicted by the cavity model as these simulations show, gives a hint on how the inclusions in the core metamaterial will need to be oriented to couple with the excited magnetic field and give rise to the required interaction with the 
resonant mode. This makes the practical realization of this setup foreseeable, since it is not necessary to build a totally isotropic metamaterial, and we predict that with a reasonable number of split-ring resonators or analogous resonant loop inclusions suitably oriented and disposed one may be able to effectively synthesize the required negative effective permeability for the core material and obtain results comparable with the model presented here. In this sense, the realization of an MNG metamaterial to be used in this setup may not represent an insurmountable task in the future. We are currently exploring and working on extensive numerical simulations of some of these possibilities. The preliminary results we have obtained so far are very encouraging in this sense and an extended manuscript on the practical realization of the MNG substrate for this setup and a proposed final antenna layout is currently in preparation.

An important issue to underline relates to the required size of the metamaterial inclusions (i.e., SRR) for this setup, which should in principle fit in the dielectric region between the patch and the ground plane. Due to the overall subwavelength size of the patch, the inclusions' dimension should be markedly lower than the wavelength of operation (in the example presented here, this implies that it should be of the order of $\lambda / 100$ or less.) Even though for the present status of the metamaterial technology this size represents a challenge for a practical realization, important and impressive advances are being made to improve the current technology in order to reduce the size of inclusions. For example, the possible use of lumped capacitances in the SRR design may be a viable way to reduce their size [37], even though this solution may not represent the most practical/optimal way for mass production of these materials in our proposed setup. So we can predict that in the near future, constructing metamaterials with very small inclusions that can be suitable for our idea may become a possibility. Regardless of needs for this technological development, the idea presented here represents an interesting possibility for using metamaterials for squeezing the patch antenna dimensions, while maintaining good matching and radiation performance.

\section{CONCLUSION}

In this contribution, we have analyzed the possibility of designing subwavelength resonant patch antennas using metamaterial blocks. After showing that the attempts proposed in the literature by other groups may not lead to efficient radiators, we have shown how a circular geometry may indeed offer interesting possibilities for choosing the proper mode of operation in these subwavelength antennas in order to obtain radiation performances comparable with those of a regular patch radiator of standard dimensions. Realistic numerical simulations, considering material dispersion, losses and the presence of the feeding network have been also presented, providing a validation of the theoretical results and showing how a practical realization is foreseeable. This may indeed open interesting venues for the design of small-scaled antennas with enhanced performance.

\section{REFERENCES}

[1] D. M. Pozar and D. H. Schaubert, Microstrip Antennas: The Analysis and Design of Microstrip Antennas and Arrays. New York: IEEE Press, 1995.
[2] J. R. James and P. S. Hall, Handbook of Microstrip Antennas. London, U.K.: Peter Peregrinus, 1989.

[3] S. Maci, G. Biffi Gentili, P. Piazzesi, and C. Salvador, "Dual-band slot-loaded patch antenna," Proc. Inst. Elect. Eng. Microw. Antennas Propag., vol. 142, no. 3, pp. 225-232, Jun. 1995.

[4] R. Porath, "Theory of miniaturized shorting-post microstrip antennas," IEEE Trans. Antennas Propag., vol. 48, no. 1, pp. 41-47, Jan. 2000.

[5] S.-S. Zhong and J.-H. Cui, "Compact circularly polarized microstrip antenna with magnetic substrate," in Proc. IEEE AP-S Int. Symp., San Antonio, TX, Jun. 2002, vol. 1, pp. 793-796.

[6] D. Psychoudakis, Y. H. Koh, J. L. Volakis, and J. H. Halloran, "Design method for aperture-coupled microstrip patch antennas on textured dielectric substrates," IEEE Trans. Antennas Propag., vol. 52, no. 10, pp. 2763-2766, Oct. 2004.

[7] C. Reilly, W. J. Chappell, J. Halloran, K. Sarabandi, J. Volakis, N. Kikuchi, and L. P. B. Katchi, "New fabrication technology for ceramic metamaterials," in Proc. IEEE AP-S Int. Symp., Jun. 16-21, 2002, vol. 2, pp. 376-379.

[8] J. L. Volakis, C.-C., Chen, M. Lee, B. Kramer, and D. Psychoudakis, "Miniaturization methods for narrowband and ultrawideband antennas," in Proc. IEEE Int. Workshop on Antenna Technology: Small Antennas and Novel Metamaterials, Mar. 7-9, 2005, pp. 119-121.

[9] G. Mumcu, K. Sertel, and J. L. Volakis, "Superdirective miniature antennas embedded within magnetic photonic crystals," in Proc. IEEE AP-S USNC/URSI Int. Symp., Washington, DC, Jul. 3-8, 2005.

[10] H. Mosallaei and K. Sarabandi, "Antenna miniaturization and bandwidth enhancement using a reactive impedance substrate," IEEE Trans. Antennas Propag., vol. 52, no. 9, pp. 2403-2414, Sep. 2004

[11] _ - "Magneto-dielectrics in electromagnetics: concept and applications," IEEE Trans. Antennas Propag., vol. 52, no. 6, pp. 1558-1567, Jun. 2004.

[12] R. W. Ziolkowski and N. Engheta, Eds., IEEE Trans. Antennas Propag., Special Issue on Metamaterials, vol. 51, pp. 2546-2750, Oct. 2003.

[13] N. Engheta, "An idea for thin subwavelength cavity resonators using metamaterials with negative permittivity and permeability," IEEE Antennas Wireless Propag. Lett., vol. 1, no. 1, pp. 10-13, 2002.

[14] A. Alù and N. Engheta, "Guided modes in a waveguide filled with a pair of single-negative (SNG), double-negative (DNG), and/or double-positive (DPS) layers," IEEE Trans. Microw. Theory Tech., vol. MTT-52, no. 1, pp. 199-210, Jan. 2004.

[15] _ _ "Polarizabilities and effective parameters for collections of spherical nano-particles formed by pairs of concentric double-negative (DNG), single-negative (SNG) and/or double-positive (DPS) metamaterial layers," J. Appl. Phys., vol. 97, May 1, 2005, 094310.

[16] A. Alù, F. Bilotti, N. Engheta, and L. Vegni, "Compact leaky-wave components using metamaterials," presented at the Proc. IEEE MTT-S Int. Microwave Symp., Long Beach, CA, Jun. 12-17, 2005.

[17] _ "Conformal omni-directional leaky-wave radiators with downtilted beams employing cylindrical metamaterial covers," in Proc. 4th European Workshop Conformal Antennas, Stockholm, Sweden, May 23-24, 2005, pp. 117-120.

[18] _ - "How metamaterials may significantly affect the wave transmission through sub-wavelength hole in a flat perfectly conducting screen," in Proc. Inst. Elect. Eng. Seminar on Metamaterials for Microwave and (Sub) Millimetre Wave Applications: Photonic Bandgap and Double Negative Designs, Components and Experiments, London, U.K., Nov. 24, 2003, pp. 11/1-11/6.

[19] _ - "Metamaterial monolayers and bilayers for enhanced transmission through a sub-wavelength aperture in a flat perfectly conducting screen," Atti della Fondazione Giorgio Ronchi, vol. LX, no. 1-2, pp. 185-190, Jan.-Apr. 2005.

[20] R. W. Ziolkowski and A. D. Kipple, "Application of double negative materials to increase the power radiated by electrically small antennas," IEEE Trans. Antennas Propag., vol. 51, no. 10, pp. 2626-2640, Oct. 2003.

[21] N. Engheta and A. Alù, "Can negative-parameter metamaterials provide high directivity for small apertures and antennas?," in Proc. USNC/CNC/URSI National Radio Science Meeting, Washington, DC, Jul. 3-8, 2005, p. 99.

[22] A. Alù and N. Engheta, "Pairing an epsilon-negative slab with a mu-negative slab: anomalous tunneling and transparency," IEEE Trans. Antennas Propag., vol. 51, no. 10, pp. 2558-2570, Oct. 2003.

[23] K. R. Carver and J. W. Mink, "Microstrip antenna technology," IEEE Trans. Antennas Propag., vol. AP-29, no. 1, pp. 2-24, Jan. 1981. 
[24] W. F. Richards, Y. T. Lo, and D. D. Harrison, "An improved theory of microstrip antennas with applications," IEEE Trans. Antennas Propag., vol. AP-29, no. 1, pp. 34-46, Jan. 1981.

[25] S. F. Mahmoud, "A new miniaturized annular ring patch resonator partially loaded by a metamaterial ring with negative permeability and permittivity," IEEE Antennas Wireless Propag. Lett., vol. 3, pp. 19-22, Nov. 1, 2004.

[26] M. E. Ermutlu and S. Tretyakov, "Patch antennas partially loaded with a dispersive backward-wave material," presented at the Proc. IEEE AP-S USNC/URSI Int. Symp., Washington, DC, Jul. 3-8, 2005.

[27] J. S. Petko and D. H. Werner, "Theoretical formulation for an electrically small microstrip patch antenna loaded with negative index materials," presented at the Proc. IEEE AP-S and USNC/URSI Int. Symp., Washington, DC, Jul. 3-8, 2005.

[28] A. Alù, F. Bilotti, N. Engheta, and L. Vegni, "Radiation properties of sub-wavelength resonant patch antennas filled with a pair of DPS, DNG, and/or SNG metamaterial blocks," presented at the Proc. IEEE AP-S and USNC/URSI Int. Symp., Washington, DC, Jul. 3-8, 2005.

[29] L. Landau and E. M. Lifschitz, Electrodynamics of Continuous Media. Oxford, U.K.: Pergamon Press, 1984.

[30] W. Rotman, "Plasma simulation by artificial dielectrics and parallelplate media," IRE Trans. Antennas Propag., pp. 82-84, 1962.

[31] J. B. Pendry, A. J. Holden, D. J. Robbins, and W. J. Stewart, "Lowfrequency plasmons in thin wire structures," J. of Physics: Condensed Matter, vol. 10, pp. 4785-4809, 1998.

[32] J. D. Jackson, Classical Electrodynamics. New York: Wiley, 1975.

[33] M. Abramowitz and I. A. Stegun, Eds., "Bessel functions and J and Y," in Handbook of Mathematical Functions With Formulas, Graphs, and Mathematical Tables. New York: Dover, 1972, pp. 358-364, 9th printing, $\$ 9.1$ in.

[34] J. B. Pendry, A. J. Holden, D. J. Robbins, and W. J. Stewart, "Magnetism from conductors and enhanced nonlinear phenomena," IEEE Trans. Microwave and Theory Techniques, vol. 47, no. 11, pp. 2075-2081, Nov. 1999

[35] P. Gay-Balmaz and O. J. F. Martin, "Efficient isotropic magnetic resonators," Appl. Phys. Lett., vol. 81, no. 5, pp. 939-941, Jul. 29, 2002.

[36] CST Microwave Studio 5.0 CST of America [Online]. Available: http:// www.cst.com

[37] S. Hrabar, J. Bartolic, and Z. Sipus, "Waveguide miniaturization using uniaxial negative permeability metamaterial," IEEE Trans. Antennas Propag., vol. 53, no. 1, pp. 110-119, Jan. 2005.

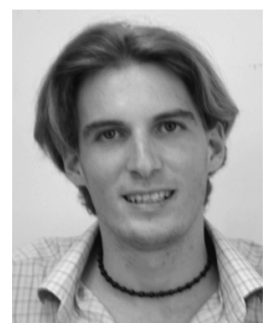

Andrea Alù (S'03) was born in Rome, Italy on September 27, 1978. He received the Laurea degree in electronics engineering (summa cum laude) and the M.S. degree from the University of Roma Tre, Rome, Italy, in 2001 and 2003, respectively, where he is currently working toward the Ph.D. degree.

Since 2002, he has been periodically visiting the University of Pennsylvania, Philadelphia, PA, where he has also developed parts of his Ph.D. research. $\mathrm{He}$ is the coauthor of more than 150 works on international books, journals, transactions and conference proceedings. His current research interests are in the areas of microwave and optical applications of complex media, metamaterials and metasurfaces, in the analysis and synthesis of planar and conformal integrated components and phased antenna arrays, in numerical methods for electromagnetics.

Mr. Alù was the recipient of an Incubic-Milton Chang Travel Award in 2006, a Young Scientist Award, presented by the International Union of Radio Science (URSI) General Assembly, in 2005, of the SUMMA Graduate Fellowship in Advanced Electromagnetics in 2004, a Young Scientist Award, presented by URSI Commission B, in 2003 and of the Raj Mittra Travel Grant Young Researcher Award in 2003. He has been awarded the second prize at the IEEE Antennas and Propagation Society (AP-S) Student Paper Contest in 2003, of which he has been finalist also in 2004 and 2005, and of other national and international awards for his research studies in electromagnetics. He is currently a student member of the IEEE Microwave Theory and Techniques Society and of the European Network of Excellence METAMORPHOSE. He serves as a reviewer of IEEE, IEE, OSA, APS Journals and Transactions.

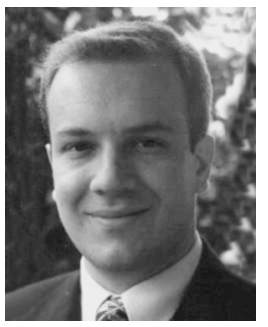

Filiberto Bilotti (S'97-M'03-SM'06) was born in Rome, Italy, on April 25, 1974. He received the Laurea (summa cum laude) and Ph.D. degrees both in electronic engineering from the University of Roma Tre, Rome, Italy, in 1998 and 2002, respectively.

Since 2002, he has been with the Department of Applied Electronics, University of Roma Tre, where he works as an Assistant Professor of electromagnetic field theory. His main research interests are in the microwave applications of complex media, metamaterials and metasurfaces; in the analysis and synthesis of planar and conformal integrated components and phased antenna arrays; in the development of improved numerical algorithms for an efficient analysis of printed antennas and circuits. He is the author of more than 175 papers on international journals and conference proceedings. Since 1999, he is a national expert of the European actions COST260 and COST284 on antenna technology and design. Since 2003, he is a technical reviewer of the European Community for scientific projects in the fields of metamaterials and antennas. Since 2004, he has been a member of the governing bodies of METAMORPHOSE, the European Network of Excellence on Metamaterials, where he acts as the coordinator of spreading activities, with particular emphasis on training events. He is also a member of the Steering Committee of the European Doctoral School on Metamaterials.

Dr. Bilotti served as a member of the Technical Program and of the Steering Committee of several national and international conferences and training events related to metamaterials, as organizer and chairman of special sessions focused on the applications of metamaterials at microwave frequencies, as a member of the Editorial Board of the Journal Metamaterials, and as a Technical Reviewer of the major international journals related to electromagnetic field theory.

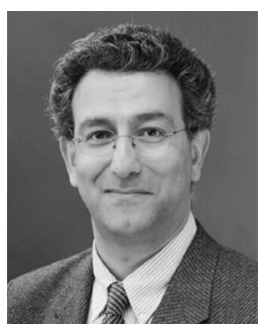

Nader Engheta (S'80-M'82-SM'89-F'96) received the B.S. degree from the University of Tehran, Iran, in 1978 and the M.S. and Ph.D. degrees from the California Institute of Technology (Caltech), in 1979 and 1982, respectively, all in electrical engineering.

After spending one year as a Postdoctoral Research Fellow at Caltech and four years as a Senior Research Scientist as Kaman Sciences Corporation's Dikewood Division in Santa Monica, CA, he joined the faculty of the University of Pennsylvania, Philadelphia, in July 1987, where he is currently the H. Nedwill Ramsey Professor of Electrical and Systems Engineering and holds an appointment in the Bioengineering Department. He is also a member of the David Mahoney Institute of Neurological Sciences. He was the Graduate Group Chair of Electrical Engineering from July 1993 to June 1997. He is the co-editor of the book Metamaterials: Physics and Engineering Explorations (Wiley-IEEE Press, 2006). His current research interests and activities span over a broad range of areas including metamaterials and plasmonics, nanooptics and nanophotonics, nanocircuits and nanostructures modeling, bio-inspired/biomimetic polarization imaging and reverse engineering of polarization vision, miniaturized antennas and nanoantennas, hyperspectral sensing, biologically-based visualization and physics of sensing and display of polarization imagery, through-wall microwave imaging, millimeter-wave lensing systems, fractional operators and fractional paradigm in electrodynamics.

Dr. Engheta is a Guggenheim Fellow, a recipient of the IEEE Third Millennium Medal, and a Fellow of the Optical Society of America. He is a member of the American Physical Society (APS), the American Association for the Advancement of Science (AAAS), Sigma Xi, Commissions B, D, and K of the U.S. National Committee (USNC) of the International Union of Radio Science (URSI), and a member of the Electromagnetics Academy. He is the ViceChair/Chair-Elect of Commission B of USNC-URSI for 2006-2008. He has received various awards and distinctions for his scholarly research contributions and teaching activities including selection as one of the 2006 Scientific American 50 leaders in science and technology, the UPS Foundation Distinguished Educator Term Chair, the Fulbright Naples Chair award, and an NSF Presidential Young Investigator (PYI) award. He was twice awarded the S. Reid Warren, Jr. Award from UPenn's School of Engineering and Applied Science, the Christian F. and Mary R. Lindback Foundation Award, and the W. M. Keck Foundation's 1995 Engineering Teaching Excellence Award. He is an Associate Editor of the IEEE ANTENNAS AND WIRELESS PROPAGATION LETTERS (2002-present), and was an Associate Editor for the IEEE TRANSACTIONS ON ANTENNA AND Propagation (1996-2001), and Radio Science (1991-1996). He was on the Editorial Board of the Journal of Electromagnetic Waves and Applications. He 
served as an IEEE Antennas and Propagation Society Distinguished Lecturer for the period 1997-99. He was the Chair (1989-91) and Vice-Chair (1988-89) of the joint chapter of the IEEE ANTENNAS AND PROPAGATION/MiCROWAVE THEORY AND TECHNIQUES in the Philadelphia Section. He served as a member of the Administrative Committee (AdCom) of the IEEE Society of Antennas and Propagation from January 2003 till December 2005. He has been a Guest Editor/Co-Editor several special issues, namely, the special issue of the Journal of Electromagnetic Waves and Applications on the topic of "Wave Interaction with Chiral and Complex Media" in 1992, part special issue of the Journal of the Franklin Institute on the topic of "Antennas and Microwaves" (from the 13th Annual Benjamin Franklin Symposium) in 1995, the special issue of the Wave Motion on the topic of "Electrodynamics in Complex Environments" in 2001, and the IEEE TRANSACTIONS ON ANTENNAS AND PROPAGATION Special Issue on Metamaterials in 2003.

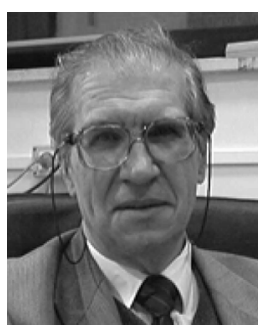

Lucio Vegni (M'73) was born in Castiglion Fiorentino, Italy, on June 20, 1943 and received the degree in electronic engineering from the University of Rome, Rome, Italy.

After a period of work at Standard Elektrik Lorenz in Stuttgart (Germany), as an Antenna Designer, he joined the Istituto di Elettronica of the University of Rome, where he was a Researcher in applied electronics. From 1976 to 1980, he was a Research Professor of applied electronics at the University of L'Aquila. From 1980 to 1985 , he became a Research
Professor of applied electronics and from 1985 to 1992 he was an Associate Professor of electromagnetic compatibility at the University of Rome "La Sapienza." Since 1992, he has been at the University of "Roma Tre", Rome, Italy, where he is currently a Full Professor of electromagnetic field theory. His research interests are in the areas of microwave and millimeter wave circuits and antennas with particular emphasis to the EMC problems. Specifically, he was active in studies of partial coherence radio links, with particular attention on multipath electromagnetic propagation effects up to 1977. Then he moved to the area of integrated microwave circuits, where he studied the electromagnetic modeling of microstrip planar circuit and antenna. In cooperation with industry he was engaged in the development of integrated microstrip antennas for satellite applications and in study of radiating system electromagnetic compatibility problems from 1985 up to 1990 . Since 1990, he is actively working on theoretical and numerical aspects of new planar antennas modeling involving unconventional materials. In these recent studies he offered new contributions to equivalent circuit representations of planar microwave components and new variational formulations for their numerical simulations. Finally, in the area of unconventional materials he gave noteworthy contributions to the study of chiral and omega grounded dielectric slab antennas. All his contributions appeared in more than 250 international papers (journals and transactions) and conferences.

Prof. Vegni is a member of the European chiral group and Italian Electrical and Electronic Society (AEI) 